\title{
Experimental Measurements of Third-Order Resonance Islands at the Photon Factory Storage Ring
}

\author{
Tsukasa Miyajima*, Yukinori Kobayashi and Kazuhito Ohmi, \\ KEK, High Energy Accelerator Research Organization, Oho 1-1, Tsukuba, Ibaraki 305-0801, Japan
}

\begin{abstract}
The coherent betatron motions after a vertical kick were examined with various field strengths of the octupole magnets near the third-order resonance line $\left(3 \nu_{y}=13\right)$, at the KEK-Photon Factory electron storage ring (PF-ring). At a particular condition, the particles were trapped in the resonance islands. The responses depended on the strength of octupole magnetic fields. As a result, we guess that the dependency of the motion in the resonance islands is generated by a slight difference of the amplitude dependent tune shift due to the octupole magnets.
\end{abstract}

\section{INTRODUCTION}

The nonlinear beam dynamics of the transverse betatron motion in circular accelerator has been studied using analytical, numerical and experimental methods $[1,2]$. Despite the progress in explanation of nonlinear phenomena, there still is a gap between analytical, numerical prediction and reality. Especially, perturbation theory is limited near a resonance. To reduce this gap, experimental nonlinearbeam-dynamics studies have become increasingly important. Recently, in the PF-ring, transverse phase space monitor system was installed [4]. We have been studied experimentally the phase space topology near resonances using this system.

In the PF-ring, a vertical instability was observed in a multi-bunch mode. To suppress transverse instabilities, four octupole magnets are installed. The octupole magnets can produce the large amplitude dependent tune shift to beam. The effect near a resonance has attracted considerable interest.

This paper concerns the study of phase space topology under various octupole field strengths near the third-order resonance.

\section{EXPERIMENTS}

\subsection{Experimental methods}

The experiment was performed in the single-bunch operation mode. The principal parameters of the PF-ring under the low-emittance optics are given in Table 1. The initial stored current was set to be about $5 \mathrm{~mA}$. The closed orbit distortions were corrected by steering dipole magnet. To measure the vertical coherent betatron motions near the third-order resonance line $\left(3 \nu_{y}=13\right)$, the initial betatron

\footnotetext{
*Email address: tsukasa@ post.kek.jp
}

Table 1: Principal parameters of the Photon Factory storage ring under the present low-emittance optics.

\begin{tabular}{lcc}
\hline Parameter & Symbol & Value \\
\hline Beam energy & $E$ & $2.5 \mathrm{GeV}$ \\
Circumference & $C$ & $187 \mathrm{~m}$ \\
Harmonic number & $h$ & 312 \\
Horizontal betatron tune & $\nu_{x}$ & 9.60 \\
Vertical betatron tune & $\nu_{y}$ & 4.28 \\
rf frequency & $f_{\mathrm{rf}}$ & $500.1 \mathrm{MHz}$ \\
Revolution period & $\tau$ & $624 \mathrm{nsec}$ \\
Emittance & $\epsilon_{x}, \epsilon_{y}$ & $36,0.36 \mathrm{nmrad}$ \\
Energy spread & $\sigma_{\epsilon}$ & 0.00073 \\
Beam size & $\sigma_{x}, \sigma_{y}$ & $0.58,0.04 \mathrm{~mm}$ \\
\hline
\end{tabular}

tunes were selected near $\left(\nu_{x}, \nu_{y}\right)=(9.60,4.33)$. The phase space monitor system consists of fast kicker magnets and turn-by-turn monitors. The fast kicker magnets provide beam with a large coherent motion. Then the initial vertical amplitude was set to be $y_{0}=3.5 \mathrm{~mm}$.

To measure the coherent betatron motion in the phase space, two BPM's at a long straight section are used. The beam position $y$ and the beam angle $y^{\prime}$ are obtained from the data of two positions.

The typical result of the experiment in which the particles was trapped in the third-order resonance island, is shown in Fig.1.

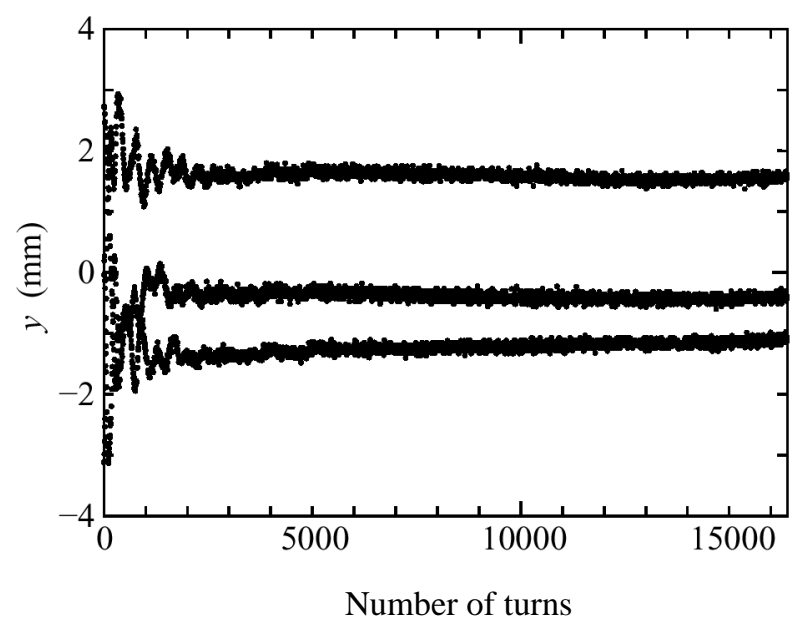

Figure 1: The vertical coherent betatron motion measured at a octupole excitation current of -1.0 A are shown as function of turn. 

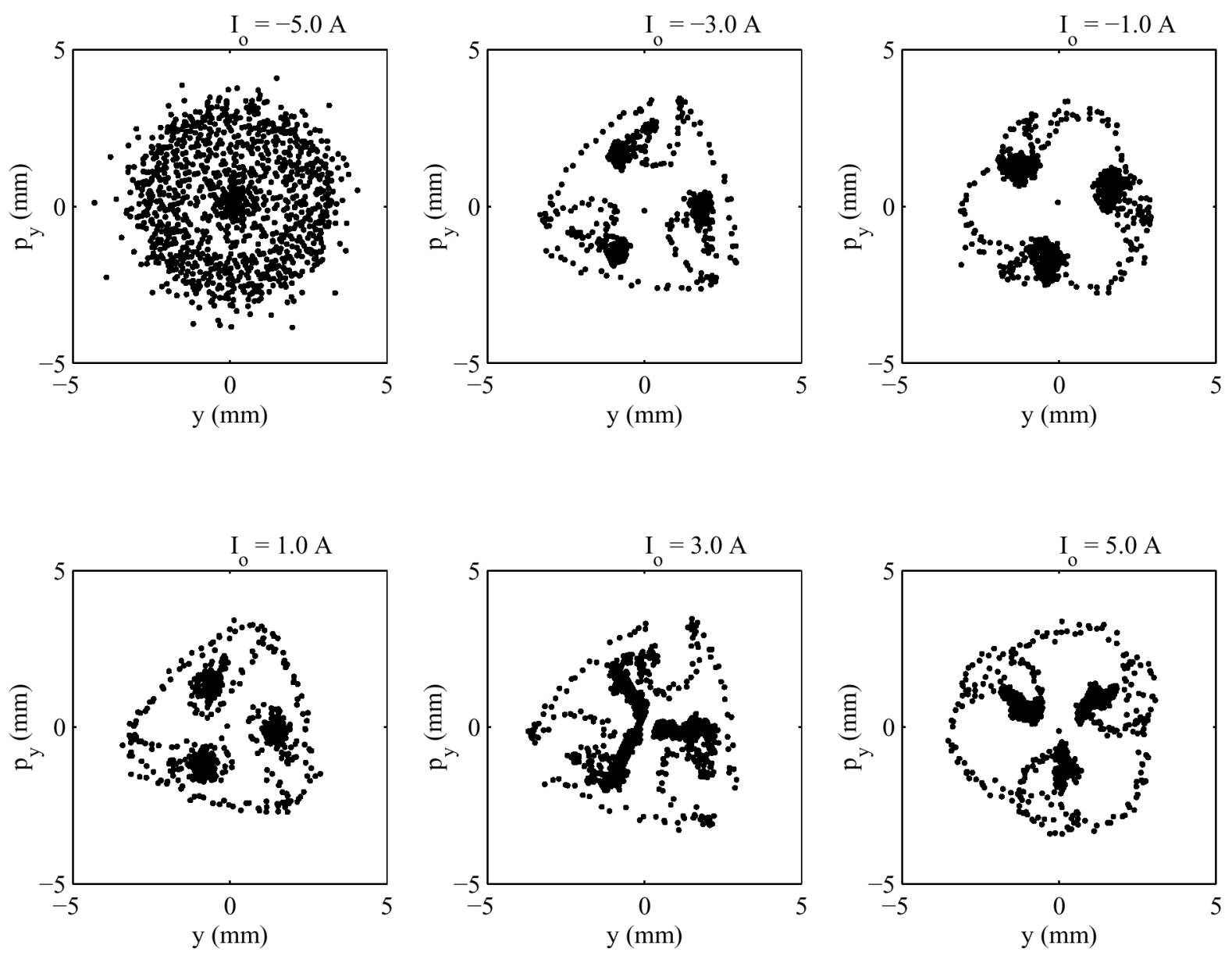

Figure 2: The Poincarè map for motion near the third-order resonance for six different octupole excitation currents: -5.0, $-3.0,-1.0,1.0,3.0$ and $5.0 \mathrm{~A}$.

\subsection{Tune shifts due to octupole magnetic field}

The octupole magnetic field gradient is given by $d^{3} B_{y} / d x^{3}=1.01 \times 10^{3} \cdot I_{o} \mathrm{~T} / \mathrm{m}^{3} / \mathrm{A}$, where $I_{o}$ is an excitation current of the octupole magnet. The tune shift due to the octupole is given by

$$
\Delta \nu_{y}=a_{1} J_{x}+a_{2} J_{y} .
$$

Where $J_{x}$ and $J_{y}$ are the horizontal and vertical action, and the coefficients $a_{1}, a_{2}$ are as follows:

$$
\begin{aligned}
a_{1} & =-\frac{1}{16 \pi} \int_{0}^{C} d s 2 K^{\prime \prime}(s) \beta_{x}(s) \beta_{y}(s), \\
a_{2} & =\frac{1}{16 \pi} \int_{0}^{C} d s K^{\prime \prime}(s) \beta_{y}^{2}(s), \\
K^{\prime \prime}(s) & =\frac{1}{B \rho}\left(\frac{\partial^{3} B_{y}}{\partial x^{3}}\right)_{x=y=0} .
\end{aligned}
$$

In this experiment, the action is $J_{x} \ll J_{y}$, because the particles are kicked to only vertical direction. Thus the vertical tune shift is given by

$$
\Delta \nu_{y} \sim a_{2} J_{y}
$$

Using the ring parameters, the vertical tune shift is estimated

$$
\Delta \nu_{y} \sim 1.95 \times 10^{3} J_{y} I_{o}
$$

\section{DATA ANALYSIS}

For particle motion in circular accelerator, the deviation from the closed orbit, $y(s)$, satisfies Hill's equation:

$$
\frac{d^{2} y}{d s^{2}}+K(s) y=\frac{\Delta B_{y}}{B \rho} .
$$

Where $K(s)$ is a function of the quadrupole strength, $B \rho$ is the magnet rigidity, and $s$ is the longitudinal particle coordinate. When the betatron motion is linear, Hill's equation can be solved using the Floquet transformation [5] to obtain the solution

$$
y(s)=\sqrt{2 \beta_{y} J_{y}} \cos \phi,
$$

where $J_{y}$ and $\phi$ are action-angle variables, $\beta_{y}$ is the vertical betatron amplitude function. Normalized momentum is given by

$$
p_{y}=\alpha_{y} y+\beta_{y} y^{\prime}
$$


where $\alpha_{y}=-1 / 2 \cdot d \beta_{y} / d y$. When linear motion is plotted in $y-p_{y}$ space, it is a circle defined by the equation,

$$
p_{y}^{2}+y^{2}=2 \beta_{y} J_{y} .
$$

The values of $\alpha_{y}$ and $\beta_{y}$, needed to transform the position variables to the normalized momentum, were determined by fitting experimental data. When the motion is linear, the phase space plot $\left(y, y^{\prime}\right)$ can be fitted to an ellipse. The Courant-Snyder parameters were determined to be $\alpha_{y}=0.186, \beta_{y}=4.40 \mathrm{~m}$ by fitting experimental data with small amplitude.

\section{MEASUREMENT RESULT AND DISCUSSION}

In order to search a condition when the particles are trapped in the resonance islands, we changed the initial betatron tunes and the vertical amplitude. Under many experimental conditions, the particles were not trapped in the island, and the coherent motions damped. At a particular condition, the particles were trapped in the island. This condition was very sensitive to the initial betatron tunes and the vertical amplitude.

Fig.2 displays a vertical phase space plot (Poincarè map) for six different octupole excitation currents. When the octupole excitation current was more than -3.0 A, the particles were trapped in the island. The motion in the phase space depended on the octupole excitation current.

The data shown in Fig.1 are plotted in $J$ - $\phi$ space in Fig.3. $\left\langle J_{y}\right\rangle$ is introduced as the position of the third-order resonance island, which is an average of the action $J_{y}$ from 9000 turns to 10000 turns. That is reason why the action $J_{y}$ approached a constant value after 9000 turns. Fig. 4 shows the average action $\left\langle J_{y}\right\rangle$ as a function of the octupole excitation current $I_{o}$. As shown Fig.4, $\left\langle J_{y}\right\rangle$ was not constant, and was minimum at $I_{o}=3.0 \mathrm{~A}$.

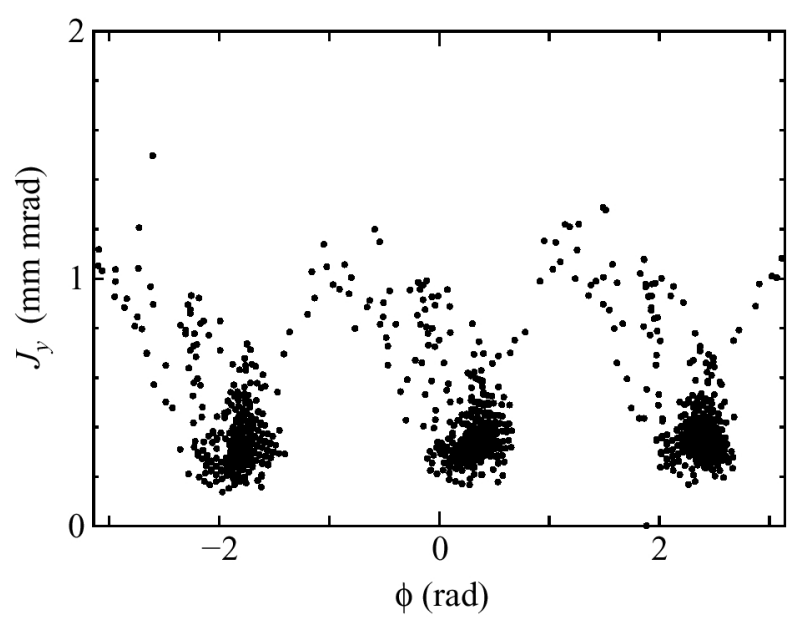

Figure 3: The Poincarè map in action-angle variables for the particles trapped in third-order resonance island at a octupole excitation current of $-1.0 \mathrm{~A}$.

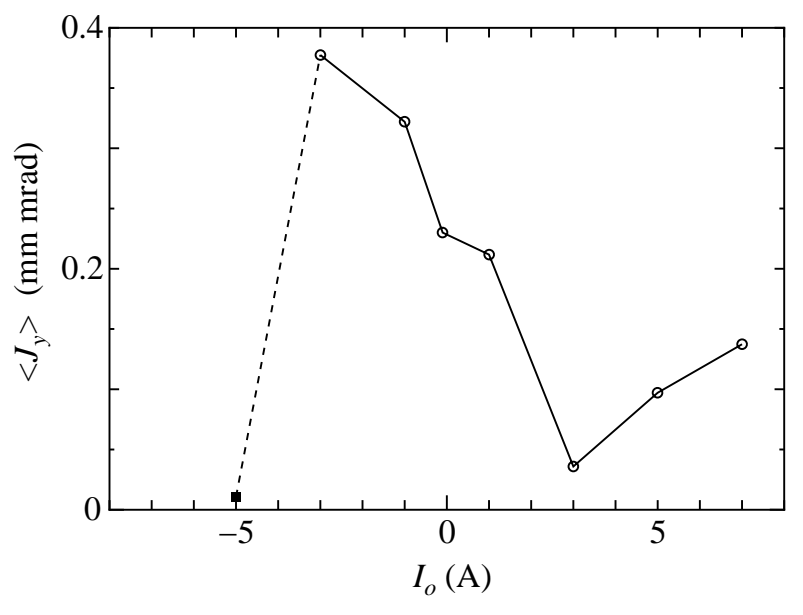

Figure 4: Average action $\left\langle J_{y}\right\rangle$ for the particles trapped in third-order resonance island for eight different octupole excitation currents: -5.0. -3.0, -1.0, -0.1, 1.0, 3.0, 5.0 and 7.0 A. For $I_{O}=-5.0$ A the particles were not trapped in the island. For $I_{o}=7.0$ A slight beam loss was observed.

In this experiment, the coherent motion after a vertical kick was examined with various field strengths of the octupole magnets near the third-order resonance line. We guess that the dependency of the motion in the resonance island is generated by a slight difference of the amplitude dependent tune shift due to the octupole magnets. Now we are going to understand the phenomena through theoretical and numerical approach in detail.

\section{ACKNOWLEDGMENT}

We wish to thank T. Kasuga and M. Kobayashi for encouraging us in this work and staff of the light source division who have been kindly supporting our experiments.

\section{REFERENCES}

[1] D.D. Caussyn et al., Phys. Rev. A 46, 7942 (1992).

[2] V. Kiselev et al., Nucl. Inst. Meth. A 406, 356 (1998).

[3] KEK Photon Factory Activity Report, 1999, edited by Y. Hori et al. (unpublished).

[4] Y. Kobayashi et al., in Proceedings of the Fifth European Particle Accelerator Conference, Sitges, 1996, pp. 1666-1668.

[5] E.D. Courant and H.S. Snyder, Ann. Phys. 3, 1 (1958). 